\title{
Soft QCD and Central Exclusive Production at LHCb
}

\author{
Marcin Kucharczyk ${ }^{* i}$ \\ Henryk Niewodniczanski Institute of Nuclear Physics PAN, Krakow, Poland \\ E-mail: marcin.kucharczykecern.ch
}

The LHCb detector, owing to its unique acceptance coverage $(2<\eta<5)$ and a precise track and vertex reconstruction, is a universal tool allowing the study of various aspects of electroweak and QCD processes, such as particle correlations or Central Exclusive Production. The recent results on the measurement of the inelastic cross section at $\sqrt{s}=13 \mathrm{TeV}$ as well as the BoseEinstein correlations of same-sign pions and kinematic correlations for pairs of beauty hadrons performed using large samples of proton-proton collision data accumulated with the LHCb detector at $\sqrt{s}=7$ and $8 \mathrm{TeV}$, are summarized in the present proceedings, together with the studies of Central Exclusive Production at $\sqrt{s}=13 \mathrm{TeV}$ exploiting new forward shower counters installed upstream and downstream of the $\mathrm{LHCb}$ detector. The results provide a valuable insight into the high-energy hadronic interactions in the forward region at the LHC.

XXVII International Workshop on Deep-Inelastic Scattering and Related Subjects - DIS2019 8-12 April, 2019

Torino, Italy

\footnotetext{
*Speaker.

${ }^{\dagger}$ On behalf of the LHCb collaboration
} 


\section{Introduction}

The LHCb detector [1] is a single-arm spectrometer fully instrumented in the forward direction, $2<\eta<5$, equipped with a high-precision tracking and particle identification systems. It provides efficient track and vertex reconstruction and an excellent particle identification at small angles with respect to the beam direction, complementary to the general purpose detectors. It proved to be a unique tool to study a broad spectrum of physics phenomena in the forward region, providing high precision results at the highest available collision energies. In addition, the forward shower counters system (HeRSCheL) [2] has been installed upstream and downstream for the Run 2 period in order to increase the rapidity coverage of the LHCb spectrometer.

The present document focuses on a precision measurement of the inelastic cross section at $\sqrt{s}=13 \mathrm{TeV}$, together with the first LHCb results on the Bose-Einstein correlations of samesign pions in proton-proton collisions at $\sqrt{s}=7 \mathrm{TeV}$ and kinematic correlations for pairs of beauty hadrons, produced in proton-proton collisions at centre-of-mass energies of 7 and $8 \mathrm{TeV}$. It also reports recent results on central exclusive particle production (CEP) for $\mathrm{J} / \psi$ and $\psi(2 \mathrm{~S})$ at $\sqrt{s}=13 \mathrm{TeV}$.

\section{Measurement of the inelastic cross section at $\sqrt{s}=13 \mathrm{TeV}$}

The LHCb measurement of the cross-section for inelastic proton-proton collisions at a centreof-mass energy of $13 \mathrm{TeV}$ was performed using a proton-proton data sample collected in 2015, corresponding to an integrated luminosity of $\sim 10.7 \mathrm{nb}^{-1}$ [3]. It was based on prompt long-lived particles inside the $\mathrm{LHCb}$ acceptance, where the event selection was based on unbiased triggers which randomly accept a small subset of all bunch crossings. The main processes contributing to the inelastic cross-section are non-diffractive, together with single and double dissociation. The measurement was primarily performed in the fiducial region with at least one prompt, long-lived charged particle with momentum $p>2 \mathrm{GeV}$ in the pseudorapidity range $2<\eta<5$, and then extrapolated to the full phase space. A particle is defined as long-lived if its lifetime is larger than $30 \mathrm{ps}$, and it is prompt if it is produced directly in the primary collision or if none of its ancestors is long-lived. The fiducial cross section was defined as a ratio of the product of the average number of interactions per event and the total number of events, devided by the integrated luminosity. The average number of interactions per event was determined from the fraction of empty events, being then corrected for detector inefficiency and wrongly reconstructed tracks. The fiducial cross-section for inelastic interactions is determined to be $\sigma_{a c c}(\sqrt{s}=13 \mathrm{TeV})=62.2 \pm 0.2($ stat. $) \pm 2.5$ (syst.) mb, where the dominant systematics comes from the luminosity measurement. The extrapolation to the full phase-space was performed using simulation, neglecting central exclusive production processes and possible interference between different contributions. The total inelastic proton-proton cross-section $\sigma_{\text {inel }}(\sqrt{s}=13 \mathrm{TeV})=75.4 \pm 3.0(\exp ) \pm 4.5($ extr $) \mathrm{mb}$, where the first uncertainty is experimental and the second one due to extrapolation. Since the publication of a measurement of the inelastic proton-proton cross-section at a centre-of-mass energy of $7 \mathrm{TeV}$ by the LHCb collaboration [4], an improved calibration of the luminosity scale has become available. An updated value of the inelastic cross-section at a centre-of-mass energy of $7 \mathrm{TeV}$ is $\sigma_{\text {inel }}(\sqrt{s}=7 \mathrm{TeV})=$ $68.7 \pm 2.1(\exp ) \pm 4.5($ extr $) \mathrm{mb}$. Figure 1 shows a comparison of the total inelastic cross-section 
measurements from proton-proton collisions at the LHC. The LHCb measurement at $\sqrt{s}=13 \mathrm{TeV}$ is in good agreement with the measurements made by the ATLAS [5] and TOTEM [6] experiments. The measured inelastic cross-section is well described by a power-law distribution.

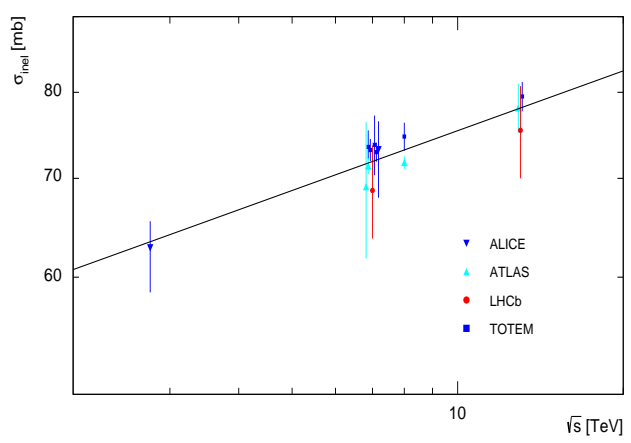

Figure 1: The total inelastic cross-section measurements from proton-proton collisions at the LHC at centreof-mass energies of 2.76, 7, 8 and $13 \mathrm{TeV}$. The error bars denote the total uncertainties. The line indicates the result from a power-law fit. Figure adopted from [3].

\section{Bose-Einstein correlations for pion pairs in proton-proton collisions at $7 \mathrm{TeV}$}

The analysis of the Bose-Einstein correlations (BEC) for the pion pairs in proton-proton collisions at $7 \mathrm{TeV}$ [7] was based on the measurement of the two-particle correlation function, $C_{2}(Q)$, commonly studied as a function of four-momenta difference $Q$, which is sensitive to the dynamic processes related to the evolution of the hadron source. It is defined as a ratio of the $Q$ distribution for same-sign pion pairs and the so-called reference distribution, which is a twoparticle distribution without the BEC effect. In the present analysis a data-driven event-mixed reference sample was used, where the pions originate from different events, so the pairs naturally do not contain quantum interference effects. The correlation function $C_{2}(Q)$ is commonly parameterised as an exponential function corresponding to the radial distribution of the static source [8], $C_{2}(Q)=N \times\left(1+\lambda \cdot e^{-R Q}\right) \times(1+\delta \cdot Q)$, where the parameter $R$, the correlation radius, can be interpreted as the radius of the spherically symmetric source of the emission volume, $N$ accounts for the overall normalisation and $\lambda$ is the chaoticity parameter, which accounts for the partial incoherence of the source. The chaoticity parameter can vary from zero, in the case of a completely coherent source, to unity for an entirely chaotic source. The $\delta$ parameter accounts for long-range correlations, e.g. related to the transverse momentum conservation. In order to account for the imperfections in the construction of the reference distribution a double ratio was used in the analysis, defined as a ratio of the correlation function in the data constructed using the event-mixed reference sample and the correlation function in the simulation without the quantum interference effects, using an event-mixed sample built with simulated events in the same way as for data. The dependence of the correlation radius and chaoticity parameter on event activity was investigated in the forward acceptance region of $2<\eta<5$ for single pions with transverse momentum $p_{T}>0.1 \mathrm{GeV} / \mathrm{c}$. Activity bins were related to the fractions of the vertex detector charged-particle multiplicity. The Coulomb corrected double ratios in three different activity bins were fitted with the exponential parametrization mentioned above. In the region of low relative momentum an en- 
hancement related to the BEC effect on the double ratio distribution for pairs of same-sign charged pions may be observed. Fig. 2 shows the dependence of the correlation parameters on event activity, where the correlation radius increases with event activity, while the chaoticity parameter is decreasing. The trends observed are in agreement with the dependencies previously measured at LEP and other LHC experiments. The $R$ and $\lambda$ parameters measured in the forward region in corresponding charged-particle multiplicity bins are slightly lower with respect to those measured by ATLAS for corresponding $p p$ interaction multiplicities [9].
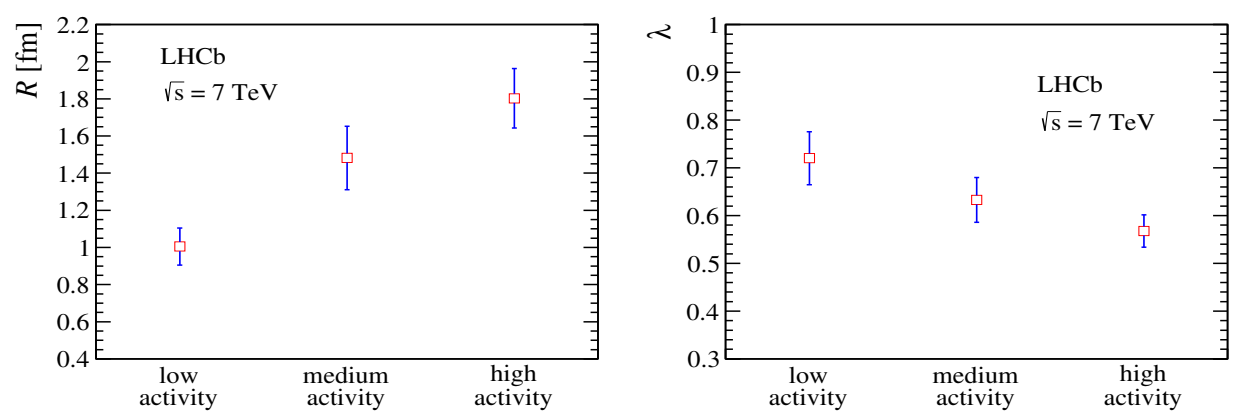

Figure 2: Correlation radius (left) and chaoticity parameter (right) as a function of event activity. Error bars indicate the sum in quadrature of the statistical and systematic uncertainties. The points are placed at the centres of the activity bins. Figure adopted from [7].

\section{Kinematic correlations for pairs of beauty hadrons at 7 and $8 \mathrm{TeV}$}

Due to a unique acceptance coverage and a detector designed to study $B$ physics, the LHCb experiment is able to provide the results on the production of heavy-flavour hadrons complementary to the central rapidity detetctors. In the present analysis the kinematic correlations for pairs of beauty hadrons were investigated using proton-proton collisions at 7 and $8 \mathrm{TeV}$. The selection was based on inclusive decays of beauty hadrons into $J / \psi$ mesons $\left(J / \psi \rightarrow \mu^{+} \mu^{-}\right)$[10], where the muons were required to have $p_{T}>500 \mathrm{MeV} / \mathrm{c}$ and $2<\eta<5$. In order to ensure a good reconstruction and trigger efficiency, both reconstructed $J / \psi$ candidates must have a good-quality vertex, a reconstructed mass in the range $3.00<m^{\mu^{+} \mu^{-}}<3.18 \mathrm{GeV} / \mathrm{c}^{2}, 2<p_{T}^{J / \psi}<25 \mathrm{GeV} / \mathrm{c}$ and $2<y^{J / \psi}<4.5$. The signal yield was determined from the fit to the two-dimensional mass distribution of muon pairs. Normalized differential cross-sections, $(1 / \sigma) \cdot(d \sigma / d v) \equiv\left(1 / N^{\text {corr }}\right) \cdot\left(\Delta N_{i}^{\text {corr }} / \Delta v_{i}\right)$, where $N^{\text {corr }}$ is a total number of efficiency-corrected signal candidates, and $\Delta N^{\text {corr }}$ is the number of efficiencycorrected signal candidates in a given $\Delta v_{i}$ bin, are determined for a set of the following kinematic variables: (i) difference in azimuthal angle of two beauty hadrons $\left|\Delta \phi^{*}\right|$; (ii) difference in pseudorapidity of two beauty hadrons $\left|\Delta \eta^{*}\right|$, where both $\phi^{*}$ and $\eta^{*}$ are determined from the direction of the vector from the primary vertex to the decay vertex of the $J / \psi$ meson; (iii) $p_{T}$ asymmetry of two $J / \psi$ mesons $A_{T} \equiv\left|\left(p_{T}^{J / \psi_{1}}-p_{T}^{J / \psi_{2}}\right) /\left(p_{T}^{J / \psi_{1}}+p_{T}^{J / \psi_{2}}\right)\right|$, (iv) mass of the $J / \psi$ pair $m^{J / \psi J / \psi} ;(v)$ transverse momentum of the $J / \psi$ pair $p_{T}^{J / \psi J / \psi}$; (vi) rapidity of the $J / \psi$ pair $y^{J / \psi J / \psi}$. By construction, the systematic uncertainty on the cross-section mostly cancels, and is much smaller than the corresponding systematic uncertainty. Figure 3 shows normalized differential production crosssections for $p_{T}^{J / \psi}>2 \mathrm{GeV} / \mathrm{c}$ compared to the PYTHIA and POWHEG generator predictions. It 
can be seen that the generator predictions describe the data reasonably well. In particular, there is a small contribution from gluon splitting at low $\left|\Delta \phi^{*}\right|$, as it is suppressed due to the large mass of the beauty quark. Both next-to-leading order and leading order predictions agree with the experimental results, thus the discriminating theory predictions is not possible with the present data. A measurement with larger statistics is needed to probe the production mechanisms.

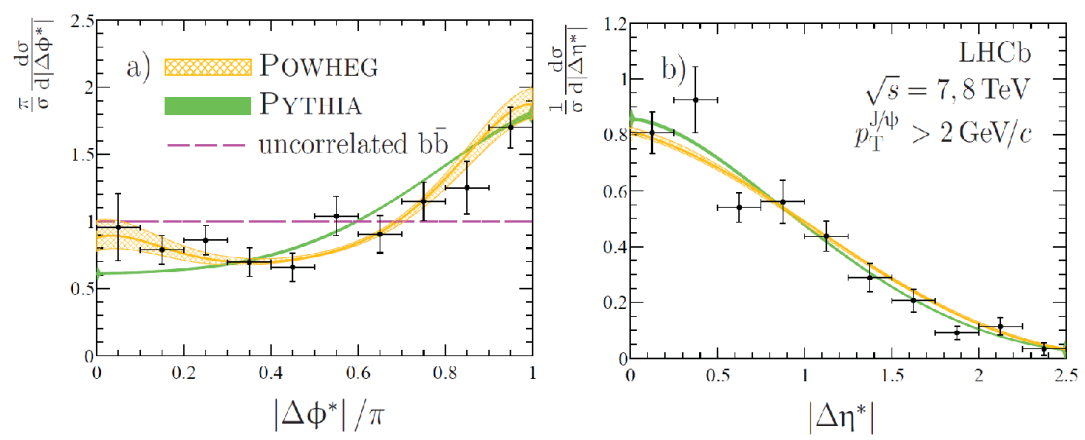

Figure 3: Normalized differential production cross-sections (points with error bars) for a) $\left|\Delta \phi^{*}\right| / \pi$ and b) $\left|\Delta \eta^{*}\right|$. Dashed magenta line on the left plot denotes the expectations for artificial data-driven model of uncorrelated $b \bar{b}$ production, orange and green areas denote the uncertainties of the POWHEG and PYTHIA predictions. Figure adopted from [10].

\section{Central Exclusive Production of $\mathrm{J} / \psi$ and $\psi(2 \mathrm{~S})$ at $13 \mathrm{TeV}$}

Measurements of exclusive $\mathrm{J} / \psi$ and $\psi(2 \mathrm{~S})$ production have been performed at $\mathrm{LHCb}$ in protonproton collisions at a centre-of-mass energy of $13 \mathrm{TeV}$ [11], employing a system of forward shower counters (HeRSCheL) [2], installed upstream and downstream of the LHCb detector. Events were selected with two muons within the pseudorapidity range $2<\eta<4.5$ and no other track in the LHCb vertex detector. In addition, a HeRSCheL veto was applied, allowing for significant reduction of backgrounds. It is a discriminating variable related to activity in HeRSCheL. The signal and background contributions were estimated from the fit by two exponential functions, where the background considered non-resonant muons, feed-downs (from exclusive production of other mesons) and undetected interacting protons. The cross-sections times branching fractions for the decays to dimuons within $2<\eta<4.5$ were measured to be $\sigma_{J / \psi \rightarrow \mu^{+} \mu^{-}}=435 \pm 18 \pm 11 \pm 17 \mathrm{pb}$ and $\sigma_{\psi(2 S) \rightarrow \mu^{+} \mu^{-}}=11.1 \pm 1.1 \pm 0.3 \pm 0.4 \mathrm{pb}$. The first uncertainty is statistical, the second one systematic, and the third is due to the luminosity determination. In addition, the cross-section times branching fraction to two muons was determined in bins of meson rapidity, showing a better agreement with the next-to-leading order predictions. A model-dependent determination of $J / \psi$ photoproduction cross-section, $\sigma(\gamma p \rightarrow J / \psi p)$ was determined from the LHCb differential crosssection measurement, assuming the power-law result from a fit to the HERA data [12] as shown in Fig. 4. A deviation from a pure power-law extrapolation of lower energy data is observed.

\section{Conclusions}

A series of measurements of soft QCD processes have been performed by LHCb, providing results in a unique forward acceptance, complementary to the results from general purpose LHC 


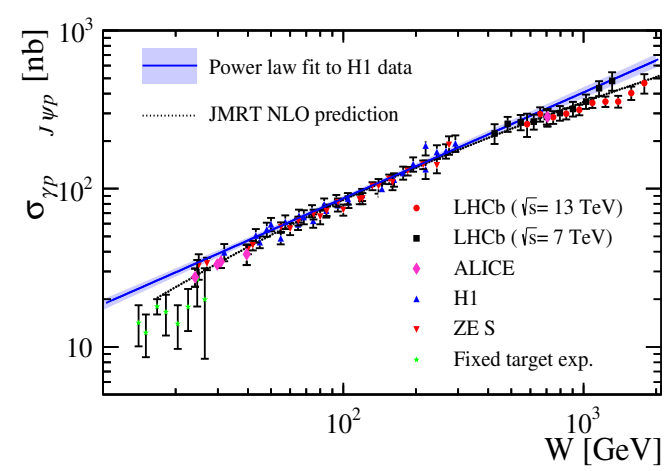

Figure 4: Compilation of photoproduction cross-section results for various experiments for J/ $\psi$. Figure adopted from [11].

detectors. The Bose-Einstein correlations between two indistinguishable pions have been observed for the first time in the forward region and the cross-section for inelastic proton-proton collisions at a centre-of-mass energy of $13 \mathrm{TeV}$ was measured. The results on kinematic $b \bar{b}$ correlations in proton-proton collisions are expected to be updated with larger statistics allowing the production mechanisms to be probed at higher sensitivity. he first measurement of exclusive $\mathrm{J} / \psi$ and $\psi(2 \mathrm{~S})$ meson production was performed on the $13 \mathrm{TeV}$ data following the installation of HeRSCheL scintillators. The results were compared to theoretical expectations, showing a better agreement with the next-to-leading order predictions. The derived cross-section for $\mathrm{J} / \psi$ photoproduction shows a deviation from a pure power-law extrapolation of $\mathrm{H} 1$ data.

\section{References}

[1] LHCb collaboration, A. A. Alves Jr. et al., JINST 3 (2008) S08005.

[2] K. Akiba et al.,JINST 13 (2018) P04017.

[3] LHCb collaboration, R. Aaij et al., JHEP 06 (2018) 100.

[4] LHCb collaboration, R. Aaij et al., JHEP 02 (2015) 029.

[5] ATLAS collaboration, M. Aaboud et al., Phys. Rev. Lett. 117 (2016) 182002.

[6] TOTEM collaboration, G. Antchev et al., CERN-EP-2017-321-v2.

[7] LHCb collaboration, R. Aaij et al., JHEP 12 (2017) 025.

[8] T. Csorgo, S. Hegyi and W. A. Zajc, Eur. Phys. J. C36 (2004) 67.

[9] ATLAS collaboration, G. Aad et al., Eur. Phys. J. C75 (2015) 466.

[10] LHCb collaboration, R. Aaij et al., JHEP 11 (2017) 030.

[11] LHCb collaboration, R. Aaij et al., JHEP 1810 (2018) 167.

[12] H1 collaboration, C. Alexa et al., Eur. Phys. J. C73 (2013) 2466. 